\title{
Final Report for Grant SC0002407
}

$8 / 15 / 09$ thru 8/14/12. The award total was $\$ 155,000$

DoE Grant SC0002407 (4th Concept Detector)

Iowa State University

John Hauptman, PI

Iowa State University, Physics

515-451-0034

hauptman@iastate.edu

Total award: $\$ 155,00015$ August $2009 \rightarrow 14$ August 2012

Unspent funds: 0

\section{Contents}

1 Introduction $\quad 1$

2 ILC: The 4th detector: a novel detector for a TeV $e^{+} e^{-}$collider $\quad 1$

2.1 The Letter of Intent . . . . . . . . . . . . . . . . . . . . . . 3

2.24 th and ILC publications $\ldots \ldots \ldots \ldots \ldots \ldots \ldots$

3 CERN: RD52 (DREAM) Dual-readout calorimetry 5

3.1 ISU accomplishments on dual-readout . . . . . . . . . . . . . . . 6

3.2 Graduate and undergraduate student summary . . . . . . . . . . . . . 8

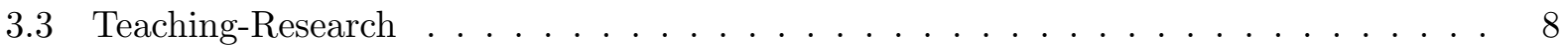

3.4 DREAM publications $\ldots \ldots \ldots \ldots \ldots \ldots$

4 Summary of Report $\quad 10$

\section{Introduction}

Department of Energy (DoE) funding through grant SC0002407 to Iowa State University have been expended on two major activities: (1) the conception, design, optimization, and promotion of the 4th Concept Detector for the International Linear Collider (ILC), and (2) research and instrumentation beam testing of dual-readout calorimeters at CERN within the DREAM collaboration.

Funds that were not fully expended during the ILC period were fully expended on support for the the DREAM collaboration at CERN on fundamental limitations to hadronic calorimetry. This project is so successful that we have been granted Project Status at the CERN Laboratory as RD52.

The following two sections describe in more detail the accomplishments made possible by this grant, SC0002407.

\section{ILC: The 4th detector: a novel detector for a TeV $e^{+} e^{-}$collider}

The 4th detector was conceived by me at the Snowmass meeting (Summer, 2005) in the wake of the first and very successful DREAM beam test at CERN in the H4 beam in the North Area. It was obvious to me that 
1. dual-readout in the simple, first attempt geometry of the DREAM module was going to be the future of hadronic calorimetry. This small module was conceived of and built by R. Wigmans (TTU) who had conceptually published its basic design at the Tucson meeting on calorimetry (1997). The first beam spill across the front of this module produced two near-Gaussian distributions of scintillation and Čerenkov light, and this test, conducted by R. Wigmans, N. Akchurin, H. Paar, and me, continued with scans of electrons up to $300 \mathrm{GeV}$ and scans with pions up to $350 \mathrm{GeV}$. Several publications in Nucl. Instrs. Meths. are the best definition of the success of the first test of this module.

2. This initial analysis of the first $\pi^{-}$beam data up to $350 \mathrm{GeV} / \mathrm{c}$ convinced me that an eventual dual-readout calorimeter without leakage, with better optics, with more Čerenkov light, and with better statistical fiber sampling would result in a hadronic calorimeter in the energy resolution range

$$
\frac{\sigma}{E} \approx \frac{20 \%}{\sqrt{E}}
$$

It appears now that that is exactly the case.

3. The three lab-supported detectors, SiD (SLAC, "Silicon Detector"), LDC (DESY, "Large Detector Concept") and GLD (KEK, "Global Large Detector") were highly similar to each other and, probably, would not work at an ILC collider (in my opinion). First, all three had identical calorimeters (designed by the CALICE group; second, two of the three had identical tracking detectors (a TPC designed by the LCTPC group); third, all three had equivalent iron flux returns for their respective magnetic fields of 3T to 5T; and fourth, the SiD group had a five-layer silicon strip tracking detector in a $5 \mathrm{~T}$ magnetic field that required "power pulsing" at $5 \mathrm{~Hz}$ in order to avoid liquid cooling.

I asked Barry Barish if I could present "a fourth" detector as an alternative to these three very similar detectors, one that would be explicitly complementary. Barish, along with Jim Brau, agreed and I gave a short 15-minute talk. It was not well received by some members of the three groups, but I was able to gather collaborators on this new " $4 \mathrm{th}$ " experiment from four regions of the world, including numerous physicists from Fermilab, the Budker Institute (including Alexandre Skrinsky), and from KEK.

I wrote a textbook on this experience, published by Wiley-VCH (Berlin) in February 2011, "Particle Physics Experiments at High Energy Colliders," starting from the basic physics of physical signals and measurements, through to the large scale thinking about magnetic fields, calorimeters and tracking systems that few students these days have a chance to think about. One comment by a $\mathrm{PhD}$ student about in the middle of his work was

"Now I see. The experimentalist connects the nut and bolt to the Feynman diagram." ( Sung Keun Park, student)

and subsequently gave a talk at the Frascati Storage Ring Conference (STOR11) on "The Evolution of Lepton Collider Detectors," 9-11 October 2011. The abstract:

The character and capabilities of lepton storage ring detectors have evolved from the simple two scintillators- in-coincidence to the very sophisticated, and future lepton collider detectors must be better by factors of 2 in calorimeter energy resolution and by factors of 10 in tracking momentum and impact parameter resolutions. I will discuss the configurations of recent and current detectors and potential future detectors at a linear electron collider (CLIC/ILC) or a Muon Collider. 
Both of these allowed me to exercise broad judgment on the physics and technologies of big instruments in high energy physics. Like the design of 4th, and as I discussed in both the book and this talk about Steinberger'a ALEPH and Richter's MAGNETIC DETECTOR, broad and wide-ranging judgments are required to build a high energy collider detector. These judgments are not restricted to technology and physics alone, but include engineering risks, personalities and egos, funding strategies, balance, and fairness and coordination within the larger group.

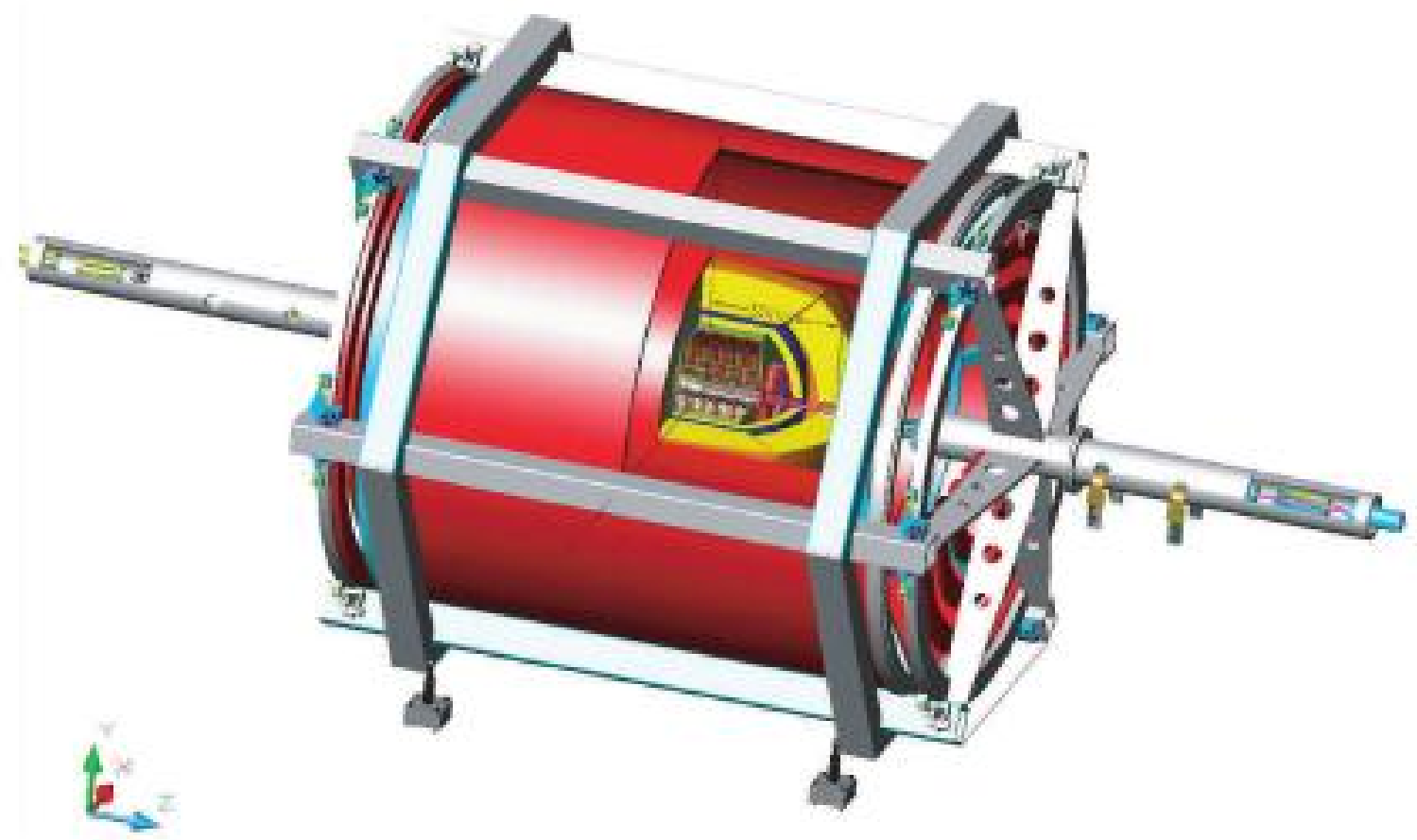

Figure 1: View of the 4th concept detector, showing the dual solenoids, the dual-readout calorimeters, and the interior tracking system.

\subsection{The Letter of Intent}

The 4th Letter of Intent is available on the ILC website and also on the ISU website (which includes the extensive engineering studies and reports that were appendices to the LoI submitted to IDAG (International Detector Advisory Group). This website is

wWw.4thconcept.org/4LoI.pdf (Letter of Intent)

www.4thconcept.org/4IDAG.pdf (Answers to questions from IDAG)

This LoI is 117 pages and was submitted to the Research Director, Sakue Yamada, on time with the other LoIs from SiD (Silicon Detector, SLAC) and ILD (International Large Detector, KEK and DESY). The Abstract to the LoI is:

We describe a general purpose detector ("4th") designed for a high-energy $e^{+} e^{-}$collider that can measure and identify with high precision all the fundamental fermions and bosons of the standard model, and thereby access all known physics processes. The 4th detector consists of four basic subsystems: (i) a pixel vertex detector for high precision 
vertex definitions, impact parameter tagging and near-beam occupancy reduction; (ii) a cluster-timing low-mass drift chamber for robust pattern recognition with over one hundred three-dimensional space-points along a track each with about $55 \mu \mathrm{m}$ resolution in $r \phi$ and $300 \mu \mathrm{m}$ resolution in $z$, and a $3.5 \%$ specific ionization measurement; (iii) high precision dual-readout calorimetry for both EM and hadronic particles, both with timehistory readout and, most importantly, hadronic energy resolution of $29 \% / \sqrt{E} \oplus 1.2 \%$ for the precision energy measurement of hadrons, jets, electrons, photons, missing momentum, and the tagging of $\mu^{ \pm}$and $\tau^{ \pm}$; and, (iv) a dual-solenoid to return the magnetic flux without iron and to provide a second field region for the inverse direction bending of muons in a gas volume to achieve high acceptance and excellent muon momentum resolution. The pixel vertex chamber, tracking and calorimeter are inside the solenoidal magnetic field.

The cluster-timing tracking chamber, the dual-readout calorimeters, and the dualsolenoid iron-free muon system are new in high energy physics, and with each we achieve superior physics performance. All four subsystems separately achieve the important scientific goal to be 2-to-10 times better than the already excellent LEP detectors, ALEPH, DELPHI, L3 and OPAL. Together these four systems are coordinated for optimal physics measurements of energy, momentum, and time. All four basic subsystems contribute to the identification of standard model partons, some in unique ways, such that consequent physics ensembles are identified with high efficiency and high purity. The cluster-timing tracking chamber is based on the successful KLOE chamber and our tested improvements to it, and the calorimeters are fully understood from comprehensive beam tests done by the DREAM collaboration.

A fifth system is the flexible and powerful root-based simulation/reconstruction/analysis/physics framework ILCroot on which all simulations of performance were achieved.

4 th is an international collaboration of over 140 people from several major laboratories (Fermilab, CERN, KEK, Cornell LNS, Budker Institute), from many universities, 29 institutions, and from four regions: 81 (Europe), 13 (Asia), 43 (US), and 3 (Fertile Crescent).

Scientifically, as an integrated detector concept, we achieve comprehensive physics capabilities that are based on new but tested instruments in high energy physics, and that put all presently conceivable physics at a future linear collider, and much speculated physics, within experimental reach.

I firmly believe that the 4th design, or something close to it, represents the most capable detector for a future lepton collider such as the ILC. As a result of this funding, many people in the high energy physics community also believe so, and we will likely revive this design and promote it for the ILC. These are the reasons, largely due to the funding of this grant:

Calorimetry: dual-readout is thoroughly beam-tested at CERN and every detail published in Nucl. Instr. Meths. The CERN laboratory has given us project status as RD52. We are approaching 1\% hadronic energy resolution at high energies, and the response function is Gaussian and at the correct energy, and all of this after a simple electron calibration.

The only other competitor for new collider calorimetry is the particle flow paradigm, but in all the talks and papers written, the actual beam response to a single pion is never shown in public. Instead, the results of a GEANT4 simulation are shown and the, even there, the "energy resolution" is barely as good as 3.5\% when the forward regions are excluded. Furtehrmore, the actual resolution is never shown, but instead an "rms-90" statistic is quoted that is computed as the rms of the response function after $10 \%$ of the distribution is excluded. 
Tracking: the SiD design calls for a 5-layer silicon tracking chamber. To keep the radiating mass down, it is proposed to use only air cooling and, to keep the heat down, to "power pulse" the silicon wafers. This means turning the power to the silicon wafers on-and-off at $5 \mathrm{~Hz}$ in a $5 \mathrm{~T}$ magnetic field. The Lorentz force will have some magnitude, depending on specific conductor placements, either large or small with respect to the supporting members. Even if the forces are small, the exact periodicity can excite mechanical harmonics of $5 \mathrm{~Hz}$. I ask periodically about testing this power pulsing on a bench. At the moment, I believe it has not been experimentally tested.

Magnetic field configuration: with the pleasant exception of ATLAS at the LHC, essentially all collider detectors have a solenoidal magnetic field for tracking and an iron flux return. With fields above $1.8 \mathrm{~T}$, iron is a problem, and the $c m d$ experiment had a few rude surprises when the field was energized; parts of the detector moved $19 \mathrm{~mm}$ and the elevator stopped working. The flux had no energy advantage to remain inside the iron and, therefore, went wherever it could find some iron to lower the stored energy of the system. This meant HF and the elevator shape. One can design this away, but iron is good only for supporting stuff. Getting rid of the iron, as detailed in the LoI, has many scientific and technological benefits.

At the moment, some high officials in the Japanese government have publicly expressed their support for Japan as the host of the ILC, meaning that Japan pays half, and the rest of the world pays half (of the expected $\$ 10 \mathrm{~B}$, or more). If Japan proceeds, and a laboratory is started and a director appointed, I will gather together the 140 participants on 4th, secure US funding at some initial level, and proceed to propose a 4th-like detector as one of the two ILC detectors. In this sense, and if we succeed, this small grant would have a huge pay-off.

\section{$2.2 \quad 4$ th and ILC publications}

1. "The Philosophy and Feasibility of Dual-Readout Calorimetry," CALOR06 Conference, 25 July 2006, Chicago.

2. "Muon identification and pion rejection in the 4th Concept", Pramana Jour. Physics, 69 December 2007, p. 1047-50.

3. "The 4th Concept Detector", Pramana Jour. Physics, 69 December 2007, p.1037-46.

4. "Particle Identification in 4th," Proc. Linear Collider Workshop, LCWS08, Chicago, IL, Nov. 2008.

5. "Dual-readout, Particle Identification, and 4th," Proc. Technology in Particle Physics, TIPP09, Nucl. Instr. Meths. A623 (2010) 237.

6. "The 4th Concept Detector for the International Linear Collider," Lepton-Photon Conference, Daegu, Korea, 13-18 August 2007, arXiv:0708.0142v2 [hep-ex] 6 Aug 2007.

\section{CERN: RD52 (DREAM) Dual-readout calorimetry}

We report on scientific work achieved from November 2011 through October 2012, and on proposed and anticipated work for the year following, November 2012 through October 2013. The work includes high precision calorimeter instrumentation with the DREAM collaboration, now officially RD52 at the CERN laboratory. In brief, ISU work includes

1. new measurements of polarization in Čerenkov light produced in a dual-readout crystal; 
2. construction and first physics use of a large volume of neutron counters in DREAM;

3. setting up a n-counter factory at CERN and in Ames;

4. new methods for making the $\mathrm{Cu}$ absorber mass in the Ames Laboratory; and,

5. new ideas for a dual-readout hadronic calorimeter with twice the mass.

Undergraduate physics majors at ISU are involved in all of this work. A press release by ISU is at

WWW. news. iastate.edu/news/2011/nov/summeratcern

Since then, I have taken two students to the Nov-Dec 2012 beam test, which was the last CERN test beam for 2.0 or 2.5 years while the LHC upgrades are being made. In addition, I still have six of these students working in my lab at ISU on several DREAM-related projects, including the manufacture of the remaining 0.5 ton of neutron counters and, most importantly, methods for building a tungsten-based dual-readout calorimeter (which would have a huge impact on the design of all collider detectors).

\subsection{ISU accomplishments on dual-readout}

We have developed new techniques in calorimetry within the DREAM collaboration, brought undergraduate physics majors into the beam tests at CERN, and contributed substantially to the overall collaboration in dual-readout calorimetry.

Neutron papers We have given several talks $[1,2]$ and published two papers $[3,4]$ on the importance of neutrons in hadronic calorimetry in DREAM. In addition, the simulations within the 4th collaboration (led by me, and this work directed by me) are consistent with the measurements of DREAM and (in a $4 \pi$ calorimeter) lead to hadronic energy resolutions of about $26 \% / \sqrt{E}$. These results (backed up by DREAM data) exceed the requirements for hadronic calorimetry at the ILC and any other future collider.

Cu extrusions and milling I initiated a small program at the Ames Laboratory (US DoE) to extrude the copper shapes needed for the "superDREAM" test module, and also directed work at Fermilab to milling grooves in Lab 3. This work was supported by Erik Ramberg (Fermilab). Both were successful, but it became clear that neither procedure could be easily extended to large $\mathrm{Cu}$ pieces.

Cerenkov fiber aluminization I organized the aluminization of the clear Cerenkov fibers by Eileen Hahn at Fermilab, also supported by Erik Ramberg. This was proposed by Wigmans as a means of measuring the depth development fluctuations by measuring the depth-of-Cerenkov-light image twice: first directly and, second, by the reflection. This measurement in the Cerenkov fibers would allow an attenuation correction in the scintillating fibers, thus canceling a constant term of order $1-2 \%$. This would also increase the Čerenkov photo-electron ( $p e$ ) yield.

Factory for making neutron counters at CERN ISU physics majors and I developed a small factory at CERN (in the scintillation lab and shop area, granted to us by Christian Joram) to produce 0.4 tonnes of neutron counters to surround any module in the DREAM tests, and eventually to surround superDREAM. We were given 1 tonne of used plastic scintillator (from the SHINE experiment, thanks to Michael Jeckel, CERN) which my students and I have re-configured into 16 neutron counters, all working in the beam, that together constitute 0.4 tons (0.6 tons 
remains to be finished, and are presently being shipped to Ames). MeV neutrons are measured in the neutron counters by their elastic scattering from the protons $n p \rightarrow n p$ in the scintillator.

It should be stated clearly that the purpose of the n-counters is to surround the eventual 6ton superDREAM module and to measure the escaping neutrons from the broken-up nuclei of the hadronic shower. This effectively measures the last fluctuation in hadronic showers and simulates an infinite calorimeter.
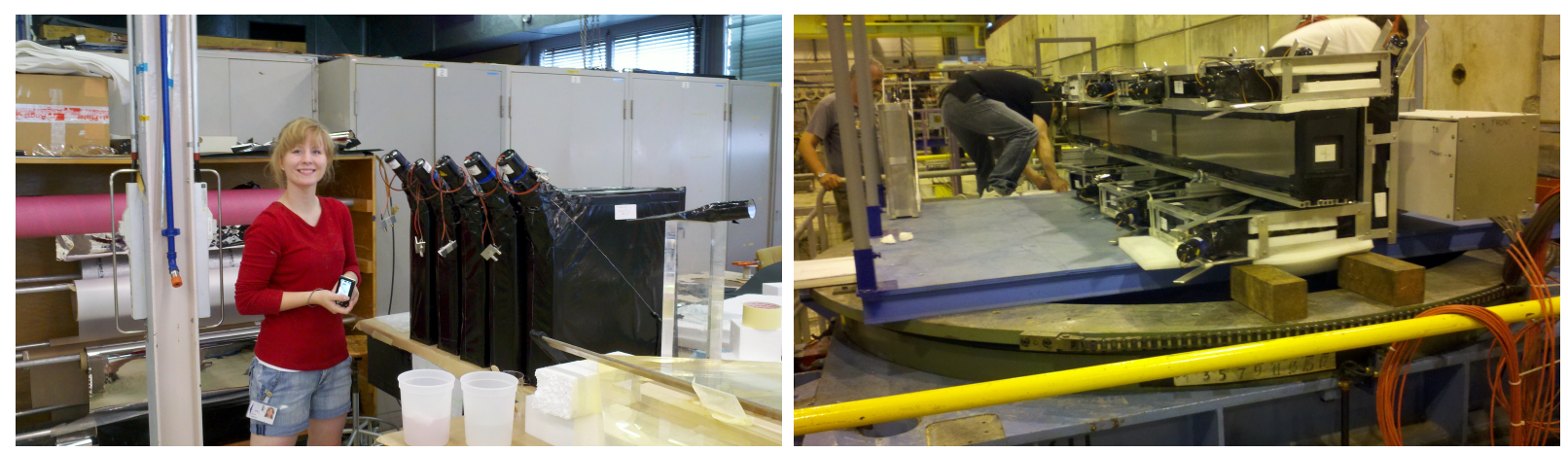

Figure 2: A first-year physics major, and one essential to the work in the CERN scintillation lab, Hanna Arnold stands in front of five finished neutron counters before they are taken out to the beam area. The n-counters can be seen positioned above, below, and behind the Pavia test module in the photo on the right. A bank of four more n-counters will be positioned on the facing side to close the volume. Next year, we will build a fixture to support these counters around any module for set of modules (superDREAM).

The 16 completed neutron-counters were put to a severe test. All n-counters were positioned around a single Pavia $\mathrm{Pb}$ module of transverse dimensions $\sim(10 \mathrm{~cm})^{2}$ and exposed to a $180 \mathrm{GeV} \pi^{-}$ beam. This single module will have huge fluctuations in leakage of 40-50\%, primarily of neutrons, hadronic debris, and some lower energy particles, limiting the energy resolution to about $\sigma / E \sim$ $30-40 \%$. Most importantly, the distribution of dual-readout pulse height shows a hadronic energy resolution $\sigma / E \approx 4.7 \%$, far lower than the $\sim 40 \%$ resolution expected without n-counters.

This demonstrates that the n-counters will be completely adequate to catch the 1-5\% fluctuations in neutron leakage from the 6-ton superDREAM module.

Rolling Cu I initiated a small program within the Ames Laboratory (funded by the Director, Alexander King) to roll $\mathrm{Cu}$ and $\mathrm{Cu}$ alloys into the fine shape needed for superDREAM. This is successful, and it is clear that we can manufacture $\mathrm{Cu}$ absorber for a full superDREAM module.

Test beam work The students worked in the beam tests: cabling, testing, and taking shifts; and built miscellaneous counters (a veto counter in the beam, an interaction target counter, and a fiber hodoscope).

Tungsten- $\mathrm{Cu}$ composites I am getting ready to develop W-Cu composite materials for a ultradense dual-readout high precision hadronic calorimeter. If successful, this will revolutionize the configuration of collider detectors, and not just the high energy colliders, but lower energy bfactories, too. The usual trade-off between tracking, calorimetry, and the solenoid will find new and highly beneficial solutions.

A photo of the first 16 (0.4 tonne) of plastic scintillator surrounding one of the Pavia Pbmoduels is shown in Fig. ??. All counters work well with signal and dynamic range up to $5 \mathrm{GeV}$ per counter. We see large neutron signals and even measure the hadronic shower shape in depth 
with these counters, and find good agreement with our other two measurements of shower depth (triangulation and light travel time).

I generally make many small contributions as work proceeds: for example, I was the one who suggested we measure polarized Čerenkov light in the BSO crystal we had (although Akchurin and Wigmans actually made the measurements); we built some beam counters; one student is building a small motor assembly to rotate the polarizer for the next round of polarization measurements; I thoroughly edit every paper (Wigmans writes them). Finally, I gave a talk on "The Evolution of Lepton Collider Detectors" at the Frascati Storage Ring Conference, STORI11. I was happy to do this, allowing me to identify the main currents of lepton collider detectors from Ada to the ILC.

\subsection{Graduate and undergraduate student summary}

Sehwook Lee was the single graduate student supported by this grant. In addition, I have brought about 13 undergraduate physics majors into the DREAM beam tests at CERN with favorable results and favorable reports from collaborators of work on shifts, cabling, building of neutron counters, and the building of ancillary beam counters.

It has been my opinion for many years that the best way to involve young students into high energy physics is to bring them directly into a working group with working physicists, put them in charge of real problems that actually count for the success of the experiment, even problems that can fail. This was done in the summer of 2011 with 10 students, and in the summer 2010 with four students (one student did both years). Of course, this received favorable publicity within the university and the physics department [5].

\subsection{Teaching-Research}

I have succeeded my whole career in involving students into productive, actual research. The secret is simple: give the student an actual problem, no fakes, no watered down problems. Almost always the student will grab this problem and, even when a little help is required from me, will do a good to excellent job on it.

\subsection{DREAM publications}

1. "Hadron and Jet Detection with a Dual-Readout Calorimeter", Akchurin, N., et al., NIM A537 (2005) 537-561.

2. "Electron Detection with a Dual-Readout Calorimeter", Akchurin, N., et al., NIM A536 (2005) 29-51.

3. "Muon Detection with a Dual-Readout Calorimeter", Akchurin, N., et al., NIM A533 (2004) 305-321.

4. "Comparison of High-Energy Electromagnetic Shower Profiles Measured with Scintillation and Cerenkov Light", Akchurin, N., et al., NIM A548 (2005) 336-354.

5. "Separation of Scintillation and Cerenkov Light in an Optical Calorimeter", Akchurin, N., et al., NIM A550 (2005) 185-200. 
6. "Measurement of the Contribution of Neutrons to Hadron Calorimeter Signals," Akchurin, N., et al., NIM A581 (2007) 643-650.

7. "Contributions of Cerenkov Light to the Signals from Lead Tungstate Crystals," Akchurin, N., et al., NIM A582 (2007) 474-483.

8. "The DREAM Project - Results and Plans,"

R. Wigmans, NIM A572 (2007) 215-217.

9. "Comparison of High-Energy Hadronic Shower Profiles Measured with Scintillation and Cerenkov Light", Akchurin, N., et al., NIM A584 (2008) 273-284.

10. "Dual-Readout Calorimetry with Lead Tungstate Crystals", Akchurin, N., et al., NIM A584 (2008) 304-318.

11. "Effects of the Temperature Dependence of the Signals from Lead Tungstate Crystals", Akchurin, N., et al., NIM A593 (2008) 530-538.

12. "Separation of Crystal Signals into Scintillation and Čerenkov Components", Akchurin, N., et al., NIM A595 (2008) 359-374.

13. "Neutron Signals for Dual-Readout Calorimetry", Akchurin, N., et al., NIM A598(2009) 422-431.

14. "Dual-Readout Calorimetry with Crystal Calorimeters", Akchurin, N., et al., NIM A598(2009) 710-721.

15. "New crystals for Dual-Readout Calorimetry," Akchurin, N., et al., NIM A604 (2009) 512-526.

16. "Dual-Readout Calorimetry with a Full-Size Electromagnetic Section," Akchurin, N., et al., NIM A610 (2009) 488-501.

17. "Optimization of Crystals for Applications in Dual-Readout Calorimetry", Akchurin, N., et al., NIM A621 (2010) 212.

18. "A Comparison of BGO and BSO Crystals Used in the Dual-Readout Mode," NIM A640 (2011) 91.

19. "Polarization as a Tool for Dual-Readout Calorimetry," NIM A638 (2011) 47.

20. "Detection of electron showers in Dual-Readout crystal calorimeters," Akchurin, N. et al., NIM A686 (2012) 125-135. 


\section{Summary of Report}

These funds were intended for the support of the 4th concept detector in competition with the two laboratory-supported concepts, SiD at SLAC and ILD at KEK/DESY, and these funds were used specifically for this purpose. When the International Detector Advisory Group (IDAG) validated $\mathrm{SiD}$ and ILD I was told that these funds would have to be redirected to dual-readout calorimetry, and they were.

If an ILC is announced by Japan, I will reform the 4th concept group, ask for modest funding to perform a slice test of all components in one beam line: silicon pixel, cluster-timing tracking, dual-readout calorimetry, and dual-solenoid iron-free muon region. This would be the only genuine slice test of any proposed detector for the ILC.

The 4th detector and the dual-readout DREAM work are highly symbiotic and have benefitted each other. DREAM has directly benefitted 4th calorimetry as the heart of this collider detector, and 4th has benefitted DREAM with the first ideas on time-history readout of all fibers, and implementation of those ideas for particle identification. 4th has also benefitted DREAM in terms of some simulations, but not powerfully so.

These two projects came together in my talk at the STORI 11 conference at Frascati on Storage Ring detectors in my talk "The Evolution of Lepton Collider Detectors", along with an accompanying paper of the same title.

\section{References}

[1] J. Hauptman, "Measurement of the neutron fraction event-by-event in DREAM," Jour. of Physics Conf. Series, 293 (2011) 012080, XIV Int'l Conf. on Calorimetry (CALOR10), Beijing, 10-14 May 2010.

[2] J. Hauptman, "Estimate of Neutrons Event-by-event in DREAM," Jour. of Physics, Conf. Series, 160 (2009) 012072; XIII Int't Conf. on Calorimetry (CALOR08).

[3] "Measurement of the contribution of neutrons to hadron calorimeter signals," N. Akchurin, et al., Nucl. Instr. Meth. A 581 (2007) 643.

[4] "Neutron signals for dual-readout calorimetry," N. Akchurin, et al., Nucl. Instr. Meth. A 598 (2009) 422-431.

[5] http://www.las.iastate.edu/newsletters/physical-sciences/s2012_students.php

[6] Internal DREAM note on details of the n-counter production and scheduling in the summer 2011.

[7] "The Evolution of Lepton Collider Detectors," Frascati Storage Ring Conference (STOR11), 9-11 October 2011. 